\title{
Impact of clinicopathological variables on laparoscopic hysterectomy complications, a tertiary center experience
}

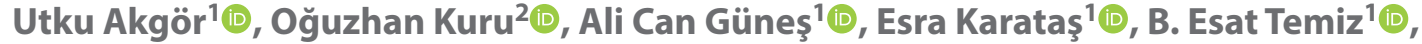

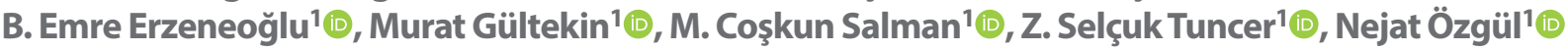 \\ ${ }^{1}$ Department of Obstetrics and Gynecology, Division of Gynecologic Oncology, Faculty of Medicine, Hacettepe University, \\ Ankara, Turkey \\ ${ }^{2}$ Department of Gynecologic Oncology, Tepecik Education and Research Hospital, Izmir, Turkey
}

\begin{abstract}
Objectives: To analyze intraoperative and postoperative complications according to Clavian-Dindo Classification (CDC) and evaluate the influence of clinicopathological features on the feasibility and safety of total laparoscopic hysterectomy (TLH) in patients that underwent surgery in a tertiary center.

Material and methods: We retrospectively reviewed the database of 469 patients that underwent surgery for patients who underwent extra facial TLH from 2013 to 2020.

Results: A total of 86 (18.3\%) peri-postoperative complications were observed. The incidence of intraoperative complications was $2 \%(n=10)$. The overall conversion rate to open surgery was $1.9 \%(n=9)$. A total of 76 postoperative complications were observed in 61 patients (14.3\%). The incidence of minor [Grade I $(n=16,3.4 \%)$ and II $(n=42,8.9 \%)$ ] and major complications [Grade III $(n=15,3.2 \%)$, IV $(n=2,0.4 \%)$ and V $(n=1,0.2 \%)$ ] were $12.3 \%$ and $3.8 \%$, respectively.

A higher BMI and performing surgery at the first step of learning are found to be associated with intraoperative and postoperative complications $(p<0.05)$. Postoperative complications related to having a history of the cesarean section, additional comorbidities, and uterine weight $\geq 300 \mathrm{~g}(\mathrm{p}<0.05)$.

Conclusions: The implementation of TLH by experienced surgeons appears to have remarkable advantages over open surgery. However, the risk factor for complications should be taken into account by surgeons in the learning curve in selecting the appropriate patient for surgery.

Key words: total laparoscopic hysterectomy; complication; learning curve; Clavian-Dindo classification
\end{abstract}

Ginekologia Polska 2022; 93, 2: 105-111

\section{INTRODUCTION}

Hysterectomy is one of the most common surgeries in gynecological procedures [1]. The vaginal hysterectomy (VH) is the preferred approach for benign cases whenever feasible. Besides, total laparoscopic hysterectomy (TLH) is a preferable alternative to open total abdominal hysterectomy (TAH) for patients in whom vaginal surgery was not suitable [2].

Traditionally, TLH has been associated with good outcomes in terms of effectively reducing symptoms, improving the quality of life and sexual functions [3]. Besides, TLH provides a bloodless dissection of anatomical spaces, better surgical precision and a view of magnified anatomy [4].
Some studies suggest that TLH appears to be associated with significantly greater rate of complications than other routes of surgical approachs $[5,6]$. Many randomized controlled trials found that laparoscopic route has a tendency to cause more bladder or ureteral injuries $[5,7,8]$. Furthermore, all these complications of TLH depend mainly on the surgeon's experience [9]. Besides this learning curve, the complications specific to laparoscopic procedures such as pneumoperitoneum, and electrosurgical instruments related complications have to be considered.

The present study aimed to examine data from consecutive TLHs. The objective was to analyze intraoperative and

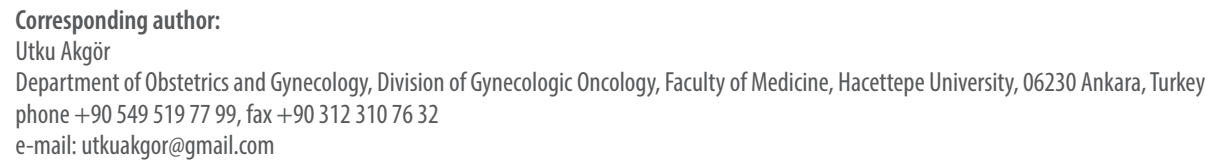


postoperative complications according to Clavian-Dindo Classification (CDC) and evaluate the influence of clinicopathological features on the feasibility and safety of TLH in 469 patients that underwent surgery in a tertiary center [10].

\section{MATERIAL AND METHODS}

The present study was approved by the institutional ethics committee of Hacettepe University, in Ankara, Turkey. Informed consent was obtained from the patients before the procedures. This research received no specific grant from any funding agency in the public, commercial, or not-for-profit sectors.

\section{Study design}

We retrospectively reviewed the database of 469 patients who underwent extrafacial laparoscopic hysterectomy from 2013 to 2020 at Hacettepe University Hospital in Ankara, Turkey. Exclusion criteria were invasive cervical cancer, endometrial and ovarian cancer requiring surgery more than extrafacial hysterectomy. All cases were performed by three obstetrics and gynecology residents training through gynecologic oncology as a subspecialist, assisted by obstetric and gynecology subspecialists. These three surgeons are well experienced in open surgical techniques but less experienced in laparoscopic surgery. The second surgeon, standard for all cases, was a fifth-year trainee of the obstetrics and gynecology residency program. With respect to hysterectomy, the second surgeon performed abdominal hysterectomy as a primary surgeon on approximately 30 cases and at least 10 laparoscopic surgical experiences as a surgical assistant. The manipulator was handled by a third-year trainee of the obstetrics and gynecology residency program in all cases.

Age, parity, body mass index (BMI), history of previous surgeries and cesarian sections, comorbidities, surgical findings, duration of surgery, hospital stay, intraoperative and postoperative complications, conversion to laparotomy and amount of blood losses were obtained from hospital records and patients files. Uterine weight, cervical length, and final histopathologies were obtained from pathology results. The 469 cases were divided into two periods to assess the surgeons' learning curve (90 cases in the first period, each surgeons' first 30 cases), and the remaining cases in the second period. The number of cases needed to achieve criteria on-level performance varies in many studies [11]. This criteria defined to be 30 cases in the present study.

The patients were assessed for intraoperative and postoperative complications (morbidity within 30 days). Postoperative complications were registered by a standardized system recorded (CDC) initially developed by Clavien in 1992 [12], and modified by Dindo in 2004 [10]. In this clas- sification the severity of the complication was graded into five groups according to the type of therapy required for treatment. Grade I complications refer to minor risk events not requiring therapy. Grade II complications refer to potentially life-threatening situations that required intervention or hospitalization. Blood transfusions and total parenteral nutrition is also included. Grade III complications refer to the need for surgical, endoscopic, or radiological intervention consist of two groups; IIIA (not under general anesthesia) and IIIB (under general anesthesia). Grade IV complications refer to life-threatening complication (including central nervous system complications) requiring Intermediate Care/Intensive Care Unit management consists of two groups; IVA (single organ dysfunction) and IVB (multiorgan dysfunction). Grade $V$ refer the death of a patient. In this study, complication was divided into minor (CDC Grade I and II) major (CDC Grade III, IV and V) categories.

Present study protocol has been approved by Institutional Review Board and Hacettepe University Ethics Committee (approval number: GO 20/501). Informed consent was not obtained from the patients due to the retrospective design of the study.

\section{Procedures}

All patients underwent pelvic examination and ultrasonography in the preoperative period. Preoperative chest X-ray, electrocardiography, and blood tests were performed. Anesthesia consultation was requested from all patients in the preoperative period. Depending on their medical history and comorbid diseases, the patients were consulted with the relevant medical departments in the preoperative period. Prophylactic antibiotics were administered $30 \mathrm{~min}$ utes before operation.

\section{Operative technique}

All procedures were performed under general anesthesia and in the dorsal lithotomy position. Clermont-Ferrand (Karl Storz Gmbh \& Co., Tuttlingen, Germany) and RUMI uterine manipulator with a Koh cup colpotomizer (Koh Colpotomizer System; Cooper Surgical, Trimbull, CT) were preferred as a uterine manipulator, and the manipulator type was randomly selected in cases regardless of any circumstances. After installing the manipulator transvaginally, the whole abdominal cavity was insufflated with $\mathrm{CO}_{2}$ with the help of a Veress needle from the abdominal umbilical point. After pneumoperitoneum creation, a $10 \mathrm{~mm}$ trochar from umblicus, and other three $5 \mathrm{~mm}$ trochars (a suprapubic and 2 laterals) were inserted. The procedure was performed with electrothermal bipolar vessel sealing device (LigaSure $^{\mathrm{TM}}$ ), dissectors, graspers, monopolar and bipolar energy modalities. The first step is dissecting the round ligaments, and opening broad ligaments. Anterior and posterior leafs of 
broad ligaments were dissected. Both ureters were checked in this step. The bladder is dissected and seperated from the uterus. The infundibulopelvic ligaments or utero-ovarian on both sides were coagulated and transected up to operation plan. If the ovaries are decided to be preserved, the fallopian were always removed. After coagulation and transection of uterin arteries and cardinal ligaments, circular colpotomy was performed with monopolar hook cautery and/or LigaSure. Specimens were retrieved vaginally, if it was possible. In inappropriate cases, manual morcellation was accomplished vaginally. The vaginal occlusion was provided to restore pneumoperitoneum. The vaginal vault was closed, laparoscopically or vaginally, with continuous vicryl no 1-0 sutures. The procedure ended with inspection and haemostasis.

All recommendations for postoperative period was similar among patients without any complications. The nasogastric tube was removed at the and of the surgery, urinary catheter was kept for six hours after surgery. Mobilization started on the day of surgery, and oral fluid intake started six hours after surgery. In cases without complications, discharge of the patients occurred after the first day of the operation. The patients were seen after the sixth week of the discharge date.

\section{Statistical analysis}

Descriptive analysis of clinicopathological characteristics was performed. Statistical differences between groups were analyzed using the Chi-square test and Student's t-test. Statistical analyses were performed using Statistical Package for the Social Sciences statistical software (version 23.0, SPSS Inc, Chicago, IL, USA). A p value of $<0.05$ was considered to indicate statistical significance.

\section{RESULTS}

The mean age of the patients was $54.7 \pm 8$.2 years. Of these 469 patients, 57 (12.1\%) were nulliparious. The mean BMI of the patients was $28.2 \pm 10.1 \mathrm{~kg} / \mathrm{m}^{2}$. One hundred and twenty-one patients ( $n=121,25.7 \%)$ had at least a history of cesarian section, and 62 patients had at least a history of abdominopelvic surgery including both open and laparoscopic surgery. Two hundred and fourty-three $(n=243$, $53 \%)$ patients had at least one medical comorbidities such as diabetes, cardiovascular disease, pulmonary disease etc (Tab. 1).

Four hundred twenty-one ( $n=421,89.7 \%$ ) of 469 patients underwent hysterectomy with adnexectomy. The mean operative time was $86 \pm 47.3$ minutes. The mean length of hospital stay was $1.6 \pm 0.4$ days. Of these patients, 9 patients (1.9\%) had been converted to laparotomy. The mean blood loss was $98.8 \pm 42.1 \mathrm{~mL}$. The surgical indications are listed in Table 1.
Table 1. Baseline characteristics, clinical features and operative outcomes of 469 patient

\begin{tabular}{|c|c|}
\hline \multicolumn{2}{|l|}{ Patients } \\
\hline Age [years] (mean) & $54.7 \pm 8.2$ \\
\hline Nulliparity: $\mathrm{n}(\%)$ & $57(12.1)$ \\
\hline BMI $\left(\mathrm{kg} / \mathrm{m}^{2}\right)$ (mean) & $28.2 \pm 10.1$ \\
\hline Previous cesarean section: $\mathrm{n}(\%)$ & $121(25.7)$ \\
\hline History of abdominopelvic surgery: $\mathrm{n}(\%)$ & $62(13.2)$ \\
\hline Comorbidities: n (\%) & $243(51.8)$ \\
\hline \multicolumn{2}{|l|}{ Operative outcomes } \\
\hline Adnexectomy (USO/BSO): n (\%) & $421(89.7)$ \\
\hline Operation time (minute) (mean) & $86 \pm 47.3$ \\
\hline Length of stay at hospital [days] & $1.6 \pm 0.4$ \\
\hline Conversion to open surgery: $\mathrm{n}(\%)$ & $9(\% 1.9)$ \\
\hline Reoperation & $7(\%$ 1.5) \\
\hline Blood loss [mL] (mean) & $98.8 \pm 42.1$ \\
\hline Intraoperative complications: Total n (\%) & $10(2)$ \\
\hline Major vascular injury: $\mathrm{n}(\%)$ & $2(0.4)$ \\
\hline Bowel injury: $\mathrm{n}(\%)$ & $1(0.2)$ \\
\hline Ureteral injury: $\mathrm{n}(\%)$ & $2(0.4)$ \\
\hline Epigastic artery injury: $\mathrm{n}(\%)$ & $3(0.6)$ \\
\hline Bladder injury: $\mathrm{n}(\%)$ & $2(0.4)$ \\
\hline \multicolumn{2}{|l|}{ Surgical indications } \\
\hline Fibroids: n (\%) & $169(36)$ \\
\hline Endometrial cancer: $\mathrm{n}(\%)$ & $49(10.4)$ \\
\hline Premalignant lesions of endometrium: $n(\%)$ & $43(9.1)$ \\
\hline Abnormal uterine bleeding & $40(8.5)$ \\
\hline Endometriosis: $\mathrm{n}(\%)$ & $39(8.3)$ \\
\hline Adenomyosis: $\mathrm{n}(\%)$ & $29(6.1)$ \\
\hline BRCA mutation & $28(5.9)$ \\
\hline Premalignant lesions of cervix: $n(\%)$ & $25(5.3)$ \\
\hline Others: $\mathrm{n}(\%)$ & $47(10)$ \\
\hline
\end{tabular}

$\mathrm{BMI}$ - body mass index; USO — unilateral salpingo-oophorectomy; BSO — bilateral salpingo-oophorectomy

The incidence of intraoperative complications was $2 \%$ $(n=10)$, and these complications included major vascular injury $(n=2,0.4 \%)$, bowel injury $(n=1,0.2 \%)$, ureteral injury $(n=2,0.4 \%)$, epigastric artery injury $(n=3,0.6 \%)$, and bladder injury $(n=2,0.4 \%)$ (Tab. 1). The intraoperative complications were more common in patients with high $\mathrm{BMI}$, endometriosis, and previous history of cesarian section. A major vascular (50\%) and the two epigastric artery injuries $(66.6 \%)$ were observed in patients with $\geq 30 \mathrm{~kg} / \mathrm{m}^{2}$. A ureter injury (50\%) was seen in a patient with endometriosis, and a bladder injury (50\%) was seen in patients with a history of cesarian section. The other intraoperative ureteral injury occurred in a patient with ipsilaterally myoma uteri of the injury side. Conversion to laparotomy was required in nine 
(1.9\%) patients, four of those were due to intraoperative complications including major vascular injury in two cases, a bowel injury, and a ureteral injury. Two conversions were due to large size uterus that hindered the movement of the uterus, and the remaining two were due to adhesions.

A total of 76 postoperative complications were observed in 64 patients (13.6\%). Among 469 patients, 54 (11.5\%) had one complication and 10 patients $(2.3 \%)$ had experienced more than one postoperative complication. All complications were categorized according to five grades of CDC as summarized in Table 2 . The incidence of minor [Grade I ( $n=16,3.4 \%)$ and II $(n=42,8.9 \%)]$ and major complications [Grade III ( $n=15,3.2 \%)$, IV $(n=2,0.4 \%)$ and V ( $n=1$, $0.2 \%)]$ were $12.3 \%$ and $3.8 \%$, respectively. Eighteen patients had major complications, seven (1.5\%) of the patients underwent reoperation including bowel perforation $(n=2)$, uterine artery bleeding $(n=2)$, ureterovaginal fistula $(n=1)$, rectovaginal fistula $(n=1)$, and vaginal vault evisceration $(n=1)$. Finally, the remaining major complications were managed with interventional radiology.

\begin{tabular}{|c|c|c|}
\hline Clavien-Dindo Score & Total, n (\%) & Complication \\
\hline Grade I & $16(3.4)$ & $\begin{array}{l}4 \text { pelvic hematomas } \\
3 \text { wound hematomas } \\
3 \text { wound infections } \\
2 \text { wound dehiscence } \\
2 \text { atelectasis } \\
2 \text { postoperative ileus }\end{array}$ \\
\hline Grade II & $42(8.9)$ & $\begin{array}{l}8 \text { urinary tract infection } \\
6 \text { blood transfusion } \\
5 \text { cuff cellulitis } \\
2 \text { deep venous thrombosis } \\
2 \text { uninary retantion } \\
2 \text { pelvic absess } \\
2 \text { bowel perforation } \\
2 \text { pelvic hematomas } \\
2 \text { ureteral injury } \\
2 \text { vaginal cuff dehiscence } \\
2 \text { pneumonia } \\
2 \text { pulmoner embolism } \\
1 \text { postoperative ileus } \\
1 \text { atelectasis } \\
1 \text { cardiac arrhythmia } \\
1 \text { myocardial infarction } \\
1 \text { mesenteric panniculitis }\end{array}$ \\
\hline Grade IIla & $3(0.6)$ & $\begin{array}{l}2 \text { pelvic absess } \\
1 \text { pelvic hematoma }\end{array}$ \\
\hline Grade IIIb & $12(2.5)$ & $\begin{array}{l}3 \text { ureteral injury } \\
3 \text { ureterovaginal fistulas } \\
2 \text { uterine artery bleeding } \\
2 \text { vesicovaginal fistulas } \\
1 \text { rectovaginal fistulas } \\
1 \text { vaginal vault evisceration }\end{array}$ \\
\hline Grade IV & $2(0.4)$ & 2 bowel perforation \\
\hline Grade V & $1(0.2)$ & 1 liver failure \\
\hline
\end{tabular}

Among postoperative major TLH complications, a rectovaginal fistula (100\%) and a ureteral injury (33.3\%) occurred after the dissection of deep infiltrating endometriosis. Two vesicovaginal fistulas (100\%) and a ureterovaginal fistula (33.3\%) were observed in patients who had a history of cesarian section. Two uterine artery bleedings (100\%) were registered in patients undergoing hysterectomy for uterine fibroids (uterine weight $\geq 300 \mathrm{~g}$ ). One bowel perforation was secondary to unwillingly cauterization was reported on the 4th day of surgery in a patient with a BMI over 30. Mortality due to liver failure was noticed in a patient with cirrhosis.

$\mathrm{BMI}$ and the period of surgery are found to be associated with total complication rates (intraoperative and postoperative) in univariate analysis $(p<0.05)$. Unlike postoperative complications, intraoperative complications are significantly associated with operative time $\geq 90$ minutes $(p<0.05$ ). Postoperative complications had a statistically significant association with having a history of cesarian section, additional comorbidities, and uterine weight $\geq 300 \mathrm{~g}(\mathrm{p}<0.05)$. Univariate analysis also showed that the age, nulliparity, adnexectomy during TLH, uterine cervical length, and uterine manipulator type were not risk factors for TLH complications in present study (Tab. 3).

The number of surgeries for each of three surgeons were reported to be 168 (35.8\%), 154 (32.8\%), 147 cases (31.4\%), respectively. There was no significant difference among the surgeons with respect to complications rate $(p=0.89)$. However, linear regression analysis reviewed a significant negative correlation between the number of surgeries performed and the rate of complications for each surgeon $(p<0.05)$.

\section{DISCUSSION}

With the preference of laparoscopic approach has the greatest increase in hysterectomy route, determining the risk factors associated with this surgical procedure is of vital importance. Laparoscopic hysterectomy is performed for a wide variety of reasons, such as significantly less pain, a shorter hospital stay, and a faster return to normal daily activities. Besides all these benefits of laparoscopic hysterectomy, many studies revealed that intraoperative and postoperative complications were more common in laparoscopic surgery $[6,13]$.

Several studies reveal a statistically significant increase in urologic complications in laparoscopic route, particularly in ureteral injury and fistula [8]. In contrary to open surgeries, the lack of certain recognition of the cervicovaginal margin in TLH cause unnecessary dissections through to the vagina. These dissections and the use of energy modalities may results with higher rates of urinary tract complications. Some may hypothesize a longer cervix and the absence of pro- 


\begin{tabular}{|c|c|c|c|c|}
\hline Variables & Intraoperative complications: $\mathbf{n}(\%)$ & p & Postoperative major complications: $\mathbf{n}(\%)$ & p \\
\hline Age & & NS & & NS \\
\hline$<60$ years & $7 / 341(2)$ & & 13/341 (3.8) & \\
\hline$\geq 60$ years & $3 / 128(2.3)$ & & $5 / 128(3.9)$ & \\
\hline Nulliparity & & NS & & NS \\
\hline Yes & $1 / 40(2.5)$ & & $2 / 40(5)$ & \\
\hline No & $9 / 429(2.1)$ & & $16 / 429(3.7)$ & \\
\hline BMI $\left[\mathrm{kg} / \mathrm{m}^{2}\right]$ & & $<0.05$ & & $<0.05$ \\
\hline$<30$ & $4 / 277(1.4)$ & & $9 / 277(3.2)$ & \\
\hline$\geq 30$ & $6 / 175(3.4)$ & & 9/175 (5.1) & \\
\hline Previous cesarean section & & NS & & $<0.05$ \\
\hline Yes & 3/121 (2.4) & & $8 / 121(6.6)$ & \\
\hline No & $7 / 348(2)$ & & $10 / 348(2.8)$ & \\
\hline Comorbidities & & NS & & $<0.05$ \\
\hline Yes & $5 / 243(2)$ & & $12 / 243(4.9)$ & \\
\hline No & $5 / 226(2.2)$ & & $6 / 226(2.6)$ & \\
\hline Adnexectomy & & - & & NS \\
\hline Yes & $10 / 421(2.3)$ & & 16/421 (3.8) & \\
\hline No & $-/ 48(0)$ & & $2 / 48(4.2)$ & \\
\hline Operation time & & $<0.05$ & & NS \\
\hline$<90 \min$ & $2 / 264(0.7)$ & & $10 / 264(3.7)$ & \\
\hline$\geq 90 \mathrm{~min}$ & $8 / 188(4.2)$ & & $8 / 188(4.2)$ & \\
\hline Uterine cervical length & & NS & & NS \\
\hline$<4 \mathrm{~cm}$ & $6 / 313(1.9)$ & & $12 / 313(3.8)$ & \\
\hline$\geq 4 \mathrm{~cm}$ & $4 / 140(2.8)$ & & $5 / 140(3.5)$ & \\
\hline Uterine weight & & NS & & $<0.05$ \\
\hline$<300 \mathrm{~g}$ & 6/354 (1.7) & & $9 / 354(2.5)$ & \\
\hline$\geq 300 \mathrm{~g}$ & 4/135 (2.9) & & $9 / 135(6.7)$ & \\
\hline Uterine manipulator & & NS & & NS \\
\hline RUMI & 2/99 (2) & & $5 / 99(5)$ & \\
\hline Clermont-Ferrand & $8 / 364(2.2)$ & & 13/364 (3.6) & \\
\hline Surgery periods & & $<0.05$ & & $<0.05$ \\
\hline First 90 cases & $4 / 90(4.4)$ & & $7 / 90(7.7)$ & \\
\hline Remaining cases & $6 / 379(1.6)$ & & $11 / 379(2.9)$ & \\
\hline \multicolumn{5}{|c|}{$\mathrm{p}<0.05$ is statistically significant } \\
\hline
\end{tabular}

NS — not significant; BMI — body mass index

lapse in nulliparous may contribute an intensive dissection. However, nulliparity and the length of cervix were not associated with any TLH complications in the present study. While the ureteral injuries were commonly identified postoperatively, the bladder injuries were commonly identified intraoperatively [14], this figure was consistent with our study. Moreover, there are studies reporting the rate of bladder injury was higher than ureteral injury. Contrary to finding, we reported higher complication rates in ureteral injury than bladder $[13,15]$.
Another variable that may lead to lower urinary tract complications is the scar tissue typically located in the lower segment of the anterior uterine wall after cesarean sections. The adhesion, due to scar tissue, leads to challenges in the dissection of vesicocervical fascia. Hence, this resulted with bladder and ureter complications in many studies $[16,17]$. Although the previous cesarean section was not directly related to intraoperative complications in the present study, there were notable effects on postoperative major complications. The presence of urinary tract complications in about 
half of postoperative major complications is a main cause of this significance. Anatomical distortions and the inability to obtain an optimal surgical field of vision secondary to causes such as increased blood loss, pelvic adhesions, and endometriosis are found to be associated with increased urinary tract injury $[8,17]$, increased length of surgery and large uterus size was the described as the other risk factor in many studies $[18,19]$. As the prior reported data, deep infiltrating endometriosis was another reason for ureter complications in the present study. Although many urinary tract complications were managed with interventional procedures or surgery, there were undetected injuries resulting in vesicovaginal $(n=2)$ and ureterovaginal $(n=3)$ fistulas in our study. The surgical interventions for fistulas performed by urologists produced excellent results in our tertiary care center.

Five of the six bowel injury cases in our study were located in the rectum. A patient with rectovaginal fistula, who had a delayed diagnosis of rectal injury, was operated due to in endometriosis with rectal involvement. Only one case occured during the initial insertion of a trocar and this intraoperative small bowel perforation was managed by conversion to open surgery. Two of the remaining rectal perforations required reoperation, and of those one had deep infiltrating endometriosis. And the early recognition of two rectal perforation caused by the monopolar energy was managed conservatively with a favorable clinical outcome.

During the course of laparoscopy in obese patients, there are difficulties such as reaching the peritoneum and maintaining pneumoperitoneum, inability to give the patient proper position during surgery due to ventilation problems, and limitation in surgical exposure. The relationship between obesity and surgical complication of TLH is controversial. Many studies revealed that the TLH complications were more frequent among patients with obesity [16, 20]. Also, there are some retrospective studies showing the similar rates of complications in TLH among obese and normal weight patients [21, 22]. This study revealed the intraoperative and postoperative major complications were more likely to seen in BMI 30 and above participants.

Having comorbidities are risk factors for surgical complications in patients who undergo TLH $[22,23]$. The present study revealed that the comorbidities were risk factors only for major postoperative complications. However, a weakness of the study is the failure to address which comorbidities are predictors. Specifying types of comorbidities might improve the quality of the preoperative assessment and treatment strategies.

The definition of large uterus is described differently in many studies, but is often used for uterine weights above 300 , or $500 \mathrm{mg}[19,24]$. In the case of large uterus, laparo- scopic hysterectomy still is not the prominent choice in most cases. However, with increasing experience, the impression of performing TLH on large uterus was strengthed. Serial studies described a significant association between TLH complications and large uterus [25]. On the other hand, this association was not found to be significant in some published studies $[19,26]$. And a prospective study advocated the uterine weight was not a predictor for TLH complications, and a higher success rate in TLH for large uterus may be associated with the surgeon's experience with only an increased operative time [27]. We determined $300 \mathrm{~g}$ as a cut-off value in the present study, and the large uterus had increased risk for postoperative complications.

The process of the translation of open surgical skills into laparoscopic surgery describe as a learning curve and it is one of the most frequently cited risk factor for laparoscopic complications. The new application of the laparoscopic technique in hysterectomy has led to an increased rate of surgical complications, even in the hands of highly experienced surgeons in open surgery. This conclusion strengthens the importance of supplying appropriate conditions for surgeons having no prior experience with laparoscopic hysterectomy to facilitate them to carry out this procedure with an optimal degree of qualification and reliability. In our first 90 cases, the whole complication rate was $24.4 \%$ and the rate of complications markedly reduced to $16.8 \%$ in remaining cases. This reflected the tendency for diminishing complications with increasing experience. This finding was consistent with previous reports $[9,28]$.

This work has numerous limitations. The first limitation was the retrospective nature of the study. The second was the sample size of the study is insufficient to conclude valid results in the heterogeneous cases with different complications. Finally, the last potential limitation was the lack of reliability for the outcomes of TLH in three surgeons that might have a different set of laparoscopic skills.

\section{CONCLUSIONS}

In conclusion, the implementation of TLH by experienced surgeons appears to have remarkable advantages over open surgery. However, risk factors for complications should be taken into account by surgeons in the learning curve in selecting the appropriate patient for surgery. Hence, the benefit and risk ratio of laparoscopic surgery in advanced cases have to be considered.

\section{Acknowledgements}

None.

\section{Conflicts of interest}

Authors declare no conflict of interest. 


\section{REFERENCES}

1. Hammer A, Rositch AF, Kahlert J, et al. Global epidemiology of hysterectomy: possible impact on gynecological cancer rates. Am J Obstet Gynecol. 2015; 213(1): 23-29, doi: 10.1016/j.ajog.2015.02.019, indexed in Pubmed: 25724402.

2. Kluivers KB. Women's preference for laparoscopic or abdominal hysterectomy. Gynecol Surg. 2009; 6(3): 223-228, doi: 10.1007/s10397-008-0455-1.

3. He H, Yang Z, Zeng D, et al. Comparison of the short-term and long-term outcomes of laparoscopic hysterectomies and of abdominal hysterectomies: a case study of 4,895 patients in the Guangxi Zhuang Autonomous Region, China. Chinese Journal of Cancer Research. 2016; 28(2): 187-196, doi: 10.21147/j.issn.1000-9604.2016.02.06.

4. Ray M, Kumar N, Kuppusamy R. The red alert zone in pelvis for radical hysterectomy: the precise anatomy and safe surgical technique. Journal of Gynecologic Surgery. 2020; 36(4): 194-197, doi: 10.1089/gyn.2019.0148.

5. Garry R, Fountain J, Mason Su, et al. The eVALuate study: two parallel randomised trials, one comparing laparoscopic with abdominal hysterectomy, the other comparing laparoscopic with vaginal hysterectomy. BMJ. 2004; 328(7432): 129, doi: 10.1136/bmj.37984.623889.F6, indexed in Pubmed: 14711749.

6. Liang C, Liu P, Cui Z, et al. Effect of laparoscopic versus abdominal radical hysterectomy on major surgical complications in women with stage IA-IIB cervical cancer in China, 2004-2015. Gynecol Oncol. 2020; 156(1): 115-123, doi: 10.1016/j.ygyno.2019.10.032, indexed in Pubmed: 31806399.

7. Aarts JWM, Nieboer TE, Johnson N. Surgical approach to hysterectomy for benign gynaecological disease. Cochrane Database of Systematic Reviews. 2015; 8: CD003677, doi: 10.1002/14651858.CD003677.pub5.

8. Benson CR, Thompson S, Li G, et al. Bladder and ureteral injuries during benign hysterectomy: an observational cohort analysis in New York State. World J Urol. 2020; 38(8): 2049-2054, doi: 10.1007/s00345-0182541-y, indexed in Pubmed: 30406476.

9. Terzi H, Biler A, Demirtas O, et al. Total laparoscopic hysterectomy: Analysis of the surgical learning curve in benign conditions. Int J Surg. 2016; 35:51-57, doi: 10.1016/j.ijsu.2016.09.010, indexed in Pubmed: 27633451.

10. Dindo D, Demartines N, Clavien PA. Classification of surgical complications: a new proposal with evaluation in a cohort of 6336 patients and results of a survey. Ann Surg. 2004; 240(2): 205-213, doi: 10.1097/01. sla.0000133083.54934.ae, indexed in Pubmed: 15273542.

11. Ghomi A, Littman P, Prasad A, et al. Assessing the learning curve for laparoscopic supracervical hysterectomy. JSLS. 2007; 11(2): 190-194, indexed in Pubmed: 17761078.

12. Clavien PA, Sanabria JR, Strasberg SM. Proposed classification of complications of surgery with examples of utility in cholecystectomy. Surgery. 1992; 111(5): 518-526, indexed in Pubmed: 1598671.

13. Hwang $\mathrm{JH}$. Urologic complication in laparoscopic radical hysterectomy: meta-analysis of 20 studies. Eur J Cancer. 2012; 48(17): 3177-3185, doi: 10.1016/j.ejca.2012.06.006, indexed in Pubmed: 22824588

14. Wong JMK, Bortoletto $P$, Tolentino J, et al. Urinary tract injury in gynecologic laparoscopy for benign indication: a systematic review. Obstet Gynecol. 2018; 131(1): 100-108, doi: 10.1097/AOG.0000000000002414, indexed in Pubmed: 29215524.

15. Uppal S, Liu JR, Kevin Reynolds R, et al. Trends and comparative effectiveness of inpatient radical hysterectomy for cervical cancer in the United
States (2012-2015). Gynecol Oncol. 2019; 152(1): 133-138, doi: 10.1016/j. ygyno.2018.09.027, indexed in Pubmed: 30424895.

16. Siedhoff MT, Carey ET, Findley AD, et al. Effect of extreme obesity on outcomes in laparoscopic hysterectomy. J Minim Invasive Gynecol. 2012; 19(6): 701-707, doi: 10.1016/j.jmig.2012.07.005, indexed in Pubmed: 23084673.

17. Mamik MM, Antosh D, White DE, et al. Risk factors for lower urinary tract injury at the time of hysterectomy for benign reasons. Int Urogynecol J. 2014; 25(8): 1031-1036, doi: 10.1007/s00192-013-2308-3, indexed in Pubmed: 24464470.

18. Brummer THI, Jalkanen J, Fraser J, et al. FINHYST, a prospective study of 5279 hysterectomies: complications and their risk factors. Human Reproduction. 2011; 26(7): 1741-1751, doi: 10.1093/humrep/der116.

19. Uccella S, Cromi A, Serati M, et al. Laparoscopic hysterectomy in case of uteri weighing $\geq 1$ kilogram: a series of 71 cases and review of the literature. J Minim Invasive Gynecol. 2014; 21(3):460-465, doi: 10.1016/j. jmig.2013.08.706, indexed in Pubmed: 24012921.

20. Morgan-Ortiz F, Soto-Pineda JM, López-Zepeda MA, et al. Effect of body mass index on clinical outcomes of patients undergoing total laparoscopic hysterectomy. Int J Gynaecol Obstet. 2013; 120(1): 61-64, doi: 10.1016/j.ijgo.2012.08.012, indexed in Pubmed: 23182797.

21. Chopin N, Malaret JM, Lafay-Pillet MC, et al. Total laparoscopic hysterectomy for benign uterine pathologies: obesity does not increase the risk of complications. Hum Reprod. 2009; 24(12): 3057-3062, doi: 10.1093/humrep/dep348, indexed in Pubmed: 19801572.

22. Twijnstra ARH, Blikkendaal MD, van Zwet EW, et al. Clinical relevance of conversion rate and its evaluation in laparoscopic hysterectomy. J Minim Invasive Gynecol. 2013; 20(1): 64-72, doi: 10.1016/j.jmig.2012.09.006, indexed in Pubmed: 23312244

23. Galvis JN, Vargas MV, Robinson HN, et al. Impact of chronic obstructive pulmonary disease on laparoscopic hysterectomy outcome. JSLS. 2019; 23(1): e2018.00089, doi: 10.4293/JSLS.2018.00089, indexed in Pubmed: 30675099.

24. Chiu LH, Chen $\mathrm{CH}, \mathrm{Tu} \mathrm{PC}$, et al. Comparison of robotic surgery and laparoscopy to perform total hysterectomy with pelvic adhesions or large uterus. J Minim Access Surg. 2015; 11(1): 87-93, doi: 10.4103/09729941.147718, indexed in Pubmed: 25598606.

25. Bonilla DJ, Mains $L$, Whitaker R, et al. Uterine weight as a predictor of morbidity after a benign abdominal and total laparoscopic hysterectomy. J Reprod Med. 2007; 52(6): 490-498, indexed in Pubmed: 17694966.

26. Terzi $H$, Hasdemir PS, Biler A, et al. Evaluation of the surgical outcome and complications of total laparoscopic hysterectomy in patients with enlarged uteruses. Int J Surg. 2016; 36(Pt A): 90-95, doi: 10.1016/j. ijsu.2016.10.024, indexed in Pubmed: 27770638.

27. Macciò A, Chiappe $G$, Kotsonis $P$, et al. Surgical outcome and complications of total laparoscopic hysterectomy for very large myomatous uteri in relation to uterine weight: a prospective study in a continuous series of 461 procedures. Arch Gynecol Obstet. 2016; 294(3): 525-531, doi: 10.1007/s00404-016-4075-0, indexed in Pubmed: 27016346.

28. Naveiro-Fuentes $M$, Rodríguez-Oliver $A$, Fernández-Parra J, et al. Effect of surgeon's experience on complications from laparoscopic hysterectomy. J Gynecol Obstet Hum Reprod. 2018; 47(2): 63-67, doi: 10.1016/j. jogoh.2017.11.004, indexed in Pubmed: 29154851. 